\title{
Regionalizing Africa: Patterns of Precipitation Variability in Observations and Global Climate Models
}

\author{
Hamada S. Badr * \\ Department of Earth and Planetary Sciences (EPS) \\ Johns Hopkins University (JHU) \\ 3400 N. Charles Street, Olin Hall, Baltimore, MD, 21218, USA \\ badr@jhu.edu \\ Amin K. Dezfuli \\ Climate and Radiation Laboratory (Code 613) \\ NASA Goddard Space Flight Center (NASA/GSFC) \\ 8800 Greenbelt Road, Greenbelt, MD 20771, USA \\ and Universities Space Research Association \\ amin.dezfuli@nasa.gov \\ Benjamin F. Zaitchik \\ Department of Earth and Planetary Sciences (EPS) \\ Johns Hopkins University (JHU) \\ 3400 N. Charles Street, Olin Hall, Baltimore, MD, 21218, USA \\ zaitchik@jhu.edu \\ Christa D. Peters-Lidard \\ Earth Sciences Division (Code 610) \\ NASA Goddard Space Flight Center (NASA/GSFC) \\ 8800 Greenbelt Road, Greenbelt, MD 20771, USA \\ christa.d.peters-lidard@nasa.gov
}

* Corresponding author at: JHU, 3400 N. Charles Street, Olin Hall, Baltimore, MD, 21218, USA. E-mail address: badr@jhu.edu (Hamada S. Badr).

Key Words: Climate Regionalization, Spatial Analysis, Spatio-temporal, Variability, Africa, Precipitation, Global Climate Models, Climate Change, GCMs, RCPs, GHGs 
Many studies have documented dramatic climatic and environmental changes that have

3 affected Africa over different timescales. These studies often raise questions regarding the spatial

4 extent and regional connectivity of changes inferred from observations, proxies, and/or derived

5 from climate models. Objective regionalization offers a tool for addressing these questions. To

6 demonstrate this potential, we present applications of hierarchical climate regionalizations of

7 Africa using observations and GCM historical simulations and future projections. First, we

8 regionalize Africa based on interannual precipitation variability using CHIRPS data for the period

9 1981-2014. A number of data processing techniques and clustering algorithms are tested to ensure

10 a robust definition of climate regions. These regionalization results highlight the seasonal and even

11 month-to-month specificity of regional climate associations across the continent, emphasizing the

12 need to consider time of year as well as research question when defining a coherent region for

13 climate analysis. CHIRPS regions are then compared to those of five GCMs for the historic period,

14 with a focus on boreal summer. Results show that some GCMs capture the climatic coherence of

15 the Sahel and associated teleconnections in a manner that is similar to observations, while other

16 models break the Sahel into uncorrelated subregions or produce a Sahel-like region of variability

17 that is spatially displaced from observations. Finally, we examine shifts in climate regions under

18 projected $21^{\text {st }}$ century climate change for different GCMs and emissions pathways. We find a

19 projected change in the coherence of the Sahel, in which the western and eastern Sahel become

20 distinct regions with different teleconnections. This pattern is most pronounced in high emissions

21 scenarios. 


\section{1. Introduction}

\section{$23 \quad 1.1$ Climate Regionalization}

Climate regionalization divides a region into homogeneous subregions based on one or

25 more climatic variables. It is a very important step in climate studies because it helps in identifying

26 the drivers of climate variability specific to each region (e.g., Dezfuli and Nicholson 2013;

27 Nicholson and Dezfuli 2013). Applying conventional geographic boundaries for climate studies is

28 often problematic because climate conditions and sensitivities can vary widely within a single

29 study area (e.g., a country, a river basin). Standard climate classification systems (e.g., Köppen

30 Climate Classification) also have limitations: (i) they represent mean climatic conditions rather

31 than temporal variability (e.g., interannual), (ii) they are not informative for identifying drivers of

32 variability, and (iii) as prescribed classification systems, rather than tools, they specify which

33 variables and variable relationships are employed in the classification, rather than allowing the

34 investigator to define characteristics of interest. Climate regionalization provides a useful

35 alternative method for defining regions when we want to: (i) unravel drivers of climate variability

36 specific to different regions and seasons (e.g., Badr et al. 2014a), and explore potential changes in

37 the future, (ii) understand the spatial distribution of climate sensitivities, or (iii) employ a flexible

38 system to understand how spatial patterns of variability differ for different climate variables (e.g.

39 air temperatures as opposed to precipitation). These features make climate regionalization

40 particularly valuable for applications that rely on identifying areas of common variability in a

41 parameter of interest; for example, managing a climatically diverse hydrologic unit in the context

42 of climate variability or change, filling in data gaps in the historic climate record, or optimizing

43 seasonal forecast systems. 
Climate regionalization applies an objective single- or multi-variate statistical technique

45 such as clustering (e.g., Burn 1989; Dezfuli 2011; Gong and Richman 1995; Isik and Singh 2008;

46 Ramachandra Rao and Srinivas 2006). Different clustering techniques have been applied in the

47 literature (Djomou et al. 2015; Herrmann and Mohr 2011; Janicot 1992; Mahe et al. 2001;

48 Nicholson 1986; Ogallo 1989), which are sensitive to conceptual approach, clustering algorithm,

49 data processing, and validation criteria. It is difficult to compare the regionalization results of

50 previous studies due to the lack of software tools that are technically designed for climate studies

51 and meet the preprocessing and postprocessing requirements. This has motivated us to develop an

52 open-source R package (Badr et al. 2014b) for Hierarchical Climate Regionalization (called,

53 "HiClimR"). Badr et al. (2015) describes the methodology and technical details of HiClimR.

54 The criteria used to interpret and validate climate regionalization may vary depending on

55 the study objectives. Here, our regionalization criteria are to find homogeneous regions that are

56 geographically contiguous, provided that the minimum region size is reasonable with respect to

57 the nature of the problem (i.e., it is consistent with general size constraints such as landscape

58 structure, data coverage and density, and known climate phenomena), and that the total number of

59 regions matches the inherent physical properties of interest (e.g., the number of regions for

60 identifying large-scale driver variability is different from dividing a country or area into regions

61 of coherent climate variability for national development). The optimum regionalization will

62 always have to involve some subjective decisions (e.g., contiguity checks and geographical

63 characteristics) since the problem is a combination of applications and statistics. However, the

64 objective criteria aim to maximize intra-regional correlations (i.e., the average correlations

65 between the region mean and all of its members) and minimize inter-regional correlations (i.e., the

66 correlations between region means). 


\subsection{Variability of African Precipitation}

Africa is a continent of climate contrasts. The general spatial pattern of variability in mean annual precipitation is widely familiar: a humid equatorial zone that includes the Congo forest,

70 transitional savannah zones as the tropics grade into the subtropics, subtropical deserts in the north

71 (Sahara) and southern (Kalahari) portions of the continent, and mid-latitude influences at northern

72 and southern extremities. Temporal precipitation variability in portions of Africa can be large and

73 is widely reported, in large part because of the social and economic impacts that hydroclimatic

74 extremes have had on the Sahel, the Horn of Africa, and several other regions.

From a climate dynamics perspective, the spatial and temporal variability of precipitation present diverse challenges for process understanding, event prediction, and climate change projection, and the climate vulnerability of many communities across Africa makes the problem particularly urgent. In general, we understand variability to be a function of synoptic to mesoscale atmospheric phenomena, including migration of the Intertropical Convergence Zone (ITCZ)

80 (Barry and Chorley 2009), the strength and location of atmospheric jets (e.g., the African Easterly

81 Jet and the Tropical Equatorial Jet) (Flohn 1964), monsoon circulations in West and East Africa,

82 and significant land-atmosphere interactions (Dickinson 1995), particularly in semi-arid zones.

83 These phenomena, in turn, are influenced by the sea surface temperatures (SST) in neighboring

84 oceans and by remote climate drivers that include the El Niño Southern Oscillation (ENSO), the

85 South Asian Monsoon, the Indian Ocean Dipole, the Atlantic Multidecadal Oscillation (AMO)

86 circulation, and many others. The relative influence of these dynamics and drivers varies in space

87 and time, and even the sign of influence of important drivers like ENSO can flip from season to 88 season or between subregions of a single country or river basin. This complexity can make it 
89 difficult to characterize drivers of variability in a systematic way, or to track spatial changes in

90 their influence over time.

91 The purpose of this study is to identify regions within Africa that are coherent with respect

92 to interannual precipitation variability. This exercise offers an example of how regionalization can

93 be applied to characterize and study patterns of climate variability. It also provides a set of

94 regionalization results that can be applied to future studies of African climate. The regionalization

95 is performed using monthly precipitation estimates from observations and outputs from global

96 climate models (GCMs). Importantly, though perhaps not surprisingly, we find that regionalization

97 of Africa is a seasonally and even monthly specific problem. For this reason we define regions for

98 each season separately, where the season is defined as a combination of months for which regions

99 are spatially stable. We also find that regions differ when using different data sources (e.g.,

100 observations versus GCM). This paper presents the results of these seasonally and dataset-specific

101 regionalizations and explores implications for understanding drivers of interannual precipitation

102 variability and for projecting climate change using different GCMs.

\section{2. Data}

105 version 2.0 (Funk et al. 2015) was used to represent the observed precipitation in the period 1981-

106 2014. The data are distributed on a $0.05^{\circ}$ grid and a primary pentad temporal resolution with the

107 availability of aggregates (dekadal and monthly) or disaggregates (daily). In this study, we used

108 CHIRPS data for Africa at the monthly temporal resolution. The data were resampled to $0.25^{\circ}$

109 resolution due to the computational and memory requirements for regionalization of the entire

110 continent of Africa. In comparison with the observational datasets available, CHIRPS data 
111 provides higher resolution, better station coverage over Africa, improved statistical approaches, 112 and updated temporal coverage.

113 Extended Reconstructed Sea Surface Temperature (ERSST) v4 (Huang et al. 2015; Liu et 114 al. 2015) monthly data $\left(2^{\circ} \times 2^{\circ}\right.$ grid resolution; 1854-present $)$ were used to test the correlation 115 patterns of each region's mean timeseries with global SST.

116 The outputs from five different GCMs (CCSM4, CNRM, GFDL, HadGEM2, and 117 MIROC5) were used to demonstrate the effectiveness of regionalization in the evaluation of GCMs

118 in terms of capturing the spatial patterns of precipitation variability and the evolution of regions in 119 response to greenhouse gas concentration as represented by coherent regions of the four 120 Representative Concentration Pathways (RCPs): RCPs, RCP2.6, RCP4.5, RCP6, and RCP8.5 121 (Moss et al. 2010).

\section{3. Methods}

123 Climate regionalization was performed to divide Africa into smaller regions that are 124 homogeneous with respect to interannual variability of precipitation for all months and 3-month 125 running average seasons. The Hierarchical Climate Regionalization (HiClimR) R package (Badr 126 et al. 2015) was used. The most appropriate clustering method was used for each case based on 127 three main criteria: homogeneity, separability, and contiguity. The homogeneity criterion 128 minimizes the within-region variability and is measured by the average correlation between the 129 region mean and its members (intra-regional correlation). The separability criterion maximizes the 130 differences between regions and is measured by the maximum correlation between the different 131 means (inter-regional correlation). The contiguity criterion visually identifies the member of each 132 region in geographical proximity not to have a region divided into distant subregions or members.

133 The method that provides higher overall homogeneity, lower inter-regional correlations, and 
134 contiguous regions is referred as "better," even if the differences are small. In general, the different

135 methods will likely generate similar regions with slightly different statistics besides the visual 136 contiguity of the regions.

137 We choose the method with better statistics and better contiguity. Specifically, results from 138 two clustering methods are presented: Ward's method (Murtagh 1983; Ward Jr 1963), which

139 minimizes the error sum of squares between all members within a region after merging, and 140 Regional Linkage (Badr et al. 2015), which minimizes the inter-regional correlation between 141 region means at each merging step. Ward's method tends to generate well-proportioned regions 142 with high homogeneity regardless the inter-regional correlations, while regional linkage 143 emphasizes separation of systematically dissimilar regions. An added advantage of regional 144 linkage is that separating systematically dissimilar records isolates noise in the dataset-i.e., 145 stations or very small clusters with completely different variability that cannot be merged into any 146 of the regions and are not correlated with other stations or clusters. The noisy stations or clusters 147 can be the result of bad data or may represent a phenomenon at a different scale (e.g., local effect). 148 For regionalization at continental scale, both forms of noise are undesirable as they are not 149 representative of the broad regions that are being defined. The isolation of that noise can also help 150 in the quality control of the data.

151 Several preprocessing options in HiClimR have been utilized to find the "optimal" regions 152 and to test the sensitivity of regionalization. Geographic masking was used to mask all stations 153 outside the continent of Africa. Grid cells with near-zero precipitation variability and/or very low 154 mean precipitation are masked out to avoid any negative impacts on the quality of regionalization. 155 The final regions are generated from detrended and standardized data to account only for the 156 interannual precipitation variability without any possible effects of the linear trend or precipitation 
157 totals. The entire CHIRPS record available at the time of analysis (1981-2014) was used for all 158 regionalizations.

159 For GCM regionalization we focus on boreal Summer (July-September, JAS). We present 160 only one season in order to make the results digestible, and we choose JAS because of its 161 importance as the primary rainy season in the Sahel. Results are presented for the Historical 162 simulations of five GCMs (CCSM4, CNRM, GFDL, HadGEM2, and MIROC5; 1960-1990). A 163 unified time period of 30 years (1960-1990) was used for all GCMs; this period is used as a 164 baseline to compare the historical and future simulations of GCMs such as the reports of 165 Intergovernmental Panel on Climate Change (IPCC). The "Historical" simulations include historic 166 observations of greenhouse gases and other external forcings but are fully coupled to the ocean 167 and are not initialized from observations; as such climatological patterns and long-term trends are 168 expected to match historical patterns but specific year-to-year and even decade-to-decade 169 variability does not align with the observed climate record.

170 The possible effects of greenhouse gas concentration on spatial patterns of interannual 171 precipitation variability are examined by performing regionalization for the four Representative 172 Concentration Pathways (RCPs: RCP2.6, RCP4.5, RCP6, and RCP8.5) simulated by CCSM4 173 model for different 30-year periods within the 2006-2100 projection, and for the entire 2006-2100

174 time period. Additionally, to compare between the regions generated from observations (1981175 2014) and CCSM4 using the same time period, we combined the CCSM4 historical simulation 176 (which runs through 2005) with the RCP 4.5 projection (2006-forward) to create a 1981-2014 177 CCSM4 record. The RCP 4.5 emissions are reasonably consistent with observation for this period, 178 and the impact of emissions trajectory on climate response on such a short time scale is small 179 relative to internal variability. CHIRPS data were regridded to match the coarse model resolution. 


\section{Results \& Discussion}

\subsection{Data Preprocessing}

HiClimR implements several features to facilitate spatio-temporal analysis applications,

183 including data filtering with geographic masking and/or mean/variance thresholds, data

184 preprocessing via detrending and standardization. These were applied as described below.

\subsubsection{Data Filtering}

Fig. 1 shows the effect of masking options on regionalization results for the interannual

187 variability of precipitation over Africa in January using Ward's clustering method, and Fig. 2

188 shows the associated clustering dendrograms for: no masking (Fig. 1A), geographic masking of

189 Africa (Fig. 1B), and masking with filtering to remove all noncontiguous subregions, including

190 Northern Africa (Fig. 1C). Filtering in this application included excluding the stations above 10N,

191 in order to focus on sub-Saharan Africa, and removing small spatially discontinuous regions, such

192 as Ethiopia in the result shown in Fig. 1C. This filtering is additional to the automatic filtering 193 techniques in HiClimR that remove stations with near-zero variance and/or very low mean defined

194 by a mean threshold (the mean threshold is typically selected as a very small fraction of the average

195 monthly total precipitation where reasonable changes in its value do not affect the regionalization

196 results; for CHIRPS data, a mean threshold of $12 \mathrm{~mm} / \mathrm{month}$ was selected). The geographic

197 masking improves the results because it excludes the artifacts introduced by Europe and small

198 islands around Africa while filtering cleans up the regions and increases overall homogeneity.

199 Fig. 2 clarifies the regionalization quality in each case. The vertical axis represents the

200 clustering height. The units of this axis depend on the clustering method, but the metric generally

201 needs to be minimized to achieve maximum intra-regional homogeneity. For example, Region 4, 202 which is mainly in Europe, has an artifact subregion in the border between Angola and Namibia 
203 that disappears after geographic masking. For Ward's clustering, the y-axis on the dendrogram is

204 the sum of squared distances within all regions and is a measure of intra-regional variance. This is

205 different from the regional-linkage method that minimizes the maximum inter-regional correlation

206 between regions as a measure of region separation. To get the "optimal" number of regions for

207 final regionalization, we need to minimize the inter-regional correlations (region separation) and

208 maximize the intra-regional correlation (region homogeneity). Hence, we add a horizontal axis on

209 the left of the dendrogram plots in Fig. 2 to show the maximum inter-regional correlation at each

210 dendrogram cut that defines the number of regions. It is clear that the overall clustering height

211 decreases when applying the geographic masking from Fig. 2A to Fig. 2B and further with filtering

212 the data from Fig. 2B to Fig. 2C, which is consistent with the regionalization qualities in Fig. 1A-

213 C.

214 We emphasize that our selection of geographic extent and masking procedure is specific to

215 the objectives of this study - to provide a stable and informative regionalization of Africa at

216 continental scale. Regionalization could just as easily be applied to a global domain, including

217 land and ocean, in order to study global scale response to major modes of climate variability, for

218 example. This would change the regionalization of Africa but might yield other insights on climate

219 variability.

220 4.1.2 Detrending and Standardization

221 Fig. 3 shows the effects of data detrending and standardization on regionalization results

222 for the interannual variability of precipitation over Africa in January using Ward's clustering

223 method. Fig. 4 shows the associated clustering dendrograms. We found that standardization had

224 no visible effect on regionalization results in this month (not shown; this may be different when

225 using a different data set or at another temporal scale, when there is large spatial variability in 
226 precipitation magnitudes), while detrending affects regions that have strong similarities or

227 differences in linear trends. In this application, detrending tends to shift the borders between

228 regions without fundamentally altering the character of the map (Fig. 3). But these shifts are

229 systematic: for example, regions 3 and 4 share a positive linear trend that tends to increase the

230 correlation between regions if detrending is not applied. These regions also show the highest inter-

231 regional correlation in the analysis, so the maximum inter-regional correlation figures shown on

232 the left side of Fig. 4 reflect higher, trend-influenced correlations between these regions in the raw

233 data (0.58) and a lower correlation when data are detrended (0.48). Note that the region merging

234 in Fig. 4 is based on Ward's method, which minimizes the variance within regions.

\section{4.2 Dissimilarity Measures}

236 The dissimilarity measure — or the nature of the temporal dimension used to regionalize

237 spatial data in HiClimR - is a crucial decision, and the choice depends on the specific application.

238 For example, regions can be generated based on interannual, intraseasonal, or daily variability or

239 on seasonal cycle. Fig. 5 shows 12 regions generated for precipitation over Africa using Ward's

240 clustering method based on the interannual variability (Annual Mean; left) and seasonality (Annual

241 Cycle; right). It is clear that the differences in seasonality don't always align with differences in

242 interannual variability: regions based on seasonality tend to align zonally, following the seasonal

243 migration of the intertropical convergence zone within tropical Africa, while those based on the

244 annual mean show both zonal and meridional structure, reflecting differing influences of remote

245 climate drivers.

\section{$246 \quad 4.3$ Monthly-Specific Regions}

247 Fig. 6 shows results of regionalization on interannual precipitation variability performed

248 separately for each month of the year. This is an optimized version of regionalization that applies 
masking, filtering, standardization and detrending, as described above. These options were

250 selected for this application based on the sensitivity analysis described in Section 4.1. Ward's

251 method provided the best regionalization results in winter months (December-March) and very

252 similar regions in May. However, it was sensitive to region size, which sometimes results in

253 dividing a large but highly homogeneous region into two or more regions. In contrast, the regional

254 linkage method is able to identify big homogenous regions like the Sahel in summer and to filter

255 out noisy data. In winter months/seasons, the regions have relatively similar size and Ward's

256 method performs well: even though the method optimizes for intra-regional homogeneity rather

257 than inter-regional separability, the correlation between regions was reasonably low, indicating the

258 separation of regions was meaningful. In summer and most transitional months, however, regional

259 linkage provides more climatically meaningful regions, while Ward's tends to split regions based

260 on size, producing multiple regions that have high inter-regional correlation. In May, both methods

261 generated very similar regions (not shown) and Ward's method was selected for the slightly better

262 overall homogeneity. Note that the numbering of regions is arbitrary; the regionalization process

263 simply distinguishes between regions based on dissimilarity measure, and the analyst must

264 interpret association of regions across datasets or regionalization methods.

265 It is clear that the spatial patterns of precipitation variability over Africa (as represented by

266 homogeneous regions in Fig. 6) are specific to calendar month. This can be used as a guideline for

267 researchers studying climate processes, as one would not want to average across months with

268 significantly different regionalization patterns in the area of interest, just as one would not want to

269 average across two poorly correlated regions in any given season. For example, for a large-scale

270 study these results suggest that January and February can be treated as a coherent season, with

271 only small changes in region boundaries in central Africa. Moving back to December or forward 
272 to March, however, regions in southern Africa begin to split and shift relative to January-February

273 in ways that merit attention before attempting to generalize across DJF or JFM in those areas. This

274 could have implications for forecasting.

275 Table 1 lists the average intra-regional correlations (between region mean and all members)

276 for all regions and the maximum inter-regional between region means. The winter months

277 (December-March) and May utilized Ward's method and their rows are highlighted in bold font.

278 All other months, especially the summer months (July-September) and months of complicated

279 variability in transition between seasons utilize the regional linkage method and its ability to isolate

280 noisy areas for removal. In other applications, where optimal quality control of the data is desired,

281 statistically isolated regions (or weather stations) could be treated in a statistical or dynamical

282 analysis to understand reasons for isolation and fill their gaps. The values of intra-regional

283 correlations in Table 1 are affected by the very high resolution and continental scale (using coarser

284 data would increase the overall homogeneity of the regions since larger grid cells are smoothed

285 averages of the included finer grid cells), which indicates that each of the regions can be divided

286 into smaller regions for finer scale applications such as hydrological analysis over one country or

287 a smaller region of interest. However, the current results target the association of sub-continental

288 regions with large-scale drivers of variability (teleconnections). The maximum inter-regional

289 correlation indicates the correlation between the most similar regions in the regionalizations. All

290 other regions have smaller or negative correlations between means.

\section{$291 \quad 4.4$ Historical vs Future (GCMs)}

292 Fig. 7 shows the regions of Africa based on interannual variability of summer (July293 September, JAS) precipitation using observations from CHIRPS (OBS; 1981-2014) and different

294 GCM historical simulations (CCM4, CNRM, GFDL, HadGEM2, and MIROC5; 1960-1990). A 
unified time period of 30 years (1960-1990) was used for all GCMs. To avoid regionalization

296 sensitivity based on clustering approach, all models are treated similarly regarding the number of

297 regions (cut-off level of the dendrogram), data thresholds (variance and mean thresholds for data

298 filtering), and preprocessing options (e.g., detrending and standardization). The regional linkage

299 method is appropriate for summer precipitation when using CHIRPS observations, the outputs

300 from the two models that exhibit similar spatial variability to observations (CCSM4 and

301 MIROC5), and CNRM. In contrast, Ward's method was relatively better for GFDL and HadGEM2

302 as it provides slightly better overall homogeneity and contiguity, and none of the clustering

303 methods or preprocessing options had a significant impact on the dominant patterns. The observed

304 spatial patterns show the Sahel as one homogeneous region with strong agreement between the

305 regions generated from observations: the Sahel region is homogeneous (intra-regional correlation

$306=\sim 0.65$ ), and independent from the other three regions. It is found that CCSM4 and MIROC5

307 have good skill in capturing the coherence of the Sahel precipitation signal in summer (strong

308 signal of unique variability over the big green region that represents the Sahel, which cannot be

309 divided into smaller subregions or the mean timeseries of subregions will be strongly correlated),

310 while the other models miss this coherence and divide the Sahel into smaller regions with

311 dissimilar interannual variability. CNRM generates random spatial patterns, GFDL simulates

312 precipitation in summer shifted in the northwestern direction and HadGEM2 divides the Sahel

313 region into eastern and western subregions. The observational analysis was repeated using CRU

314 in place of CHIRPS; results were similar and are not shown.

315 The robustness of Sahel region in Summer (JAS) was examined using regionalization of

316 different observational data sources (CHIRPS, CRU, and GPCC) and a variety of homogeneity

317 checks such as correlation patterns between the region mean and precipitation over Africa. All 
318 data sources yield similar spatial patterns and identify the Sahel as a single coherent region.

319 Increasing the number of clusters or changing the clustering algorithm divides Sahel into two or

320 more "very similar" regions (i.e., the inter-regional correlations between the subregions are high

321 meaning that we need to merge them into one region).

322 Fig. 8 shows the regionalizations of Africa based on interannual variability of JAS

323 precipitation in 1960-1990 for six different ensemble members of CCSM4. All ensemble members

324 identify the Sahel and East Africa as homogeneous regions, consistent with the observations. The

325 known West African dipole mode is also detected in all cases. The differences primarily appear in

326 West Equatorial Africa, which has a strong intrinsic heterogeneity with respect to interannual

327 variability (Balas et al. 2007; Dezfuli and Nicholson 2013).

328 To compare between the regions generated from observations (1981-2014) and CCSM4

329 using the same time period, we combine the CCSM4 historical simulation (which runs through

330 2005) with the RCP 4.5 projection (2006-forward) to create a 1981-2014 CCSM4 record. The RCP

3314.5 emissions are reasonably consistent with observation for this period, and the impact of

332 emissions trajectory on climate response on such a short time scale is small relative to internal

333 variability. CHIRPS data were regridded to match the coarse model resolution. It is found the

334 spatial patterns of interannual precipitation variability captured by CCSM4 in 1981-2014 (Fig. 9),

335 especially over the Sahel, are very similar to the unified period (1960-1990) in Fig. 7. In the next

336 sections, we focus on CCSM4 for the GCM and use the unified period to facilitate comparison 337 with CHIRPS.

338 Fig. 10 shows the correlation patterns of the four regions generated based on the interannual

339 variability of JAS precipitation using CHIRPS observations with global ERSST for the mean

340 timeseries of the region and Fig. 11 shows the correlations patterns from CCSM4 with its own 
341 SST (both 1981-2014). Similarities and differences between observed regions and CCSM4 regions

342 are strongly related to SST teleconnections. Region 3 of CCSM4, which is the largest difference

343 between the observational and GCM-based regionalization, shows a positive correlation with the

344 SSTs over the ENSO region. However, such an association does not exist for any of the

345 observation-based regions; it appears to be a false ENSO teleconnection that exists only in the

346 model. For region 1, which corresponds to the Sahel in both model and simulations, the correlation

347 with North Atlantic SST (i.e., Atlantic Multidecadal Oscillation or AMO) is markedly different

348 and even slightly reversed for CCSM4 (Fig. 11) relative to observations (Fig. 10). In addition, the

349 model shows significant Region 1 correlations with Gulf of Guinea SSTs that are not seen in

350 observations. Together these differences suggest that Sahel sensitivity to Atlantic Ocean variability

351 is substantially different in CCSM4 than it is in observation. Region 2 of CCSM4 in Fig. 11, along

352 the Guinean coast, shows weaker correlation with SSTs of adjacent waters than the observations.

353 These agreements or disagreements between the regions of the observed and simulated

354 precipitation raise many interesting questions about the underlying mechanisms and skills of the

355 model dynamics and need further investigation.

356 The regions do not respond to unique climate drivers, but they have different variability in

357 response to a set of large-scale and local drivers. Regionalization helps in identifying regions with

358 coherent variability that are different from each other's. The climate drivers of a specific region

359 can then be identified, which are greatly improved over the commonly used geographic boxes that

360 could mix inhomogeneous regions and create unrealistic variability and associated drivers (e.g.,

361 the mean of an area defined by a box without performing regionalization to test its homogeneity

362 may include different -or perhaps opposite-variability that can ruin the analysis).

\section{$363 \quad 4.5$ Climate Projections}


Fig. 12 shows the possible effect of greenhouse gas concentrations on spatial patterns of

365 interannual precipitation variability as represented by coherent regions simulated by CCSM4

366 model for the period 2006-2100 for each of the four Representative Concentration Pathways

367 (RCPs: RCP2.6, RCP4.5, RCP6, and RCP8.5). In these simulations the Sahel falls into two

368 regions: regions 1 and 2 in Fig. 12. As radiative forcing increases (RCP2.6 through RCP8.5),

369 region 2 shrinks and region 1 grows: essentially, there is a shift from a coherent Sahel band that

370 stretches almost across the continent (region 2 in RCP2.6) to two distinct eastern and western Sahel

371 regions in higher emissions scenarios. The changes in Equatorial West Africa and East Africa

372 (regions 3 and 4) are small. We note that these 2006-2100 regionalization results average across

373 variability in regions that is observed in shorter periods of analysis. The evolution of regions for

374 the four climate projections examined at three 30-years different simulation periods (2010-2040,

375 2040-2070, and 2070-2100) suggest that the spatial patterns vary throughout the 21 st century,

376 perhaps as a response to changes in global SST-rainfall relationships, but that there is a gradual

377 trend towards a split between the eastern and western Sahel (not shown).

378 Fig. 13 and Fig. 14, respectively, show the correlation patterns of CCSM4 JAS region 1

379 (western Sahel) and region 2 (eastern Sahel) precipitation from all RCPs with the corresponding

380 SST field for the entire simulation period (2006-2100). Teleconnections in low emissions scenarios

381 (RCP2.6, RCP4.5) are, as expected, more similar to Sahel teleconnections in historical simulations.

382 This is particularly evident for the eastern Sahel (region 2; Fig. 14), which approximately

383 corresponds to the unified Sahel in historical simulations (Fig. 9). For all emissions scenarios,

384 region 1 shows weaker correlation with global SST patterns than region 2 does, but the strength of

385 these connections increases as the region expands under higher emissions. Region 2 shows strong 
correlations with the ENSO region and with the Indian Ocean under all RCPs, though the

387 relationship is strongest for low emissions.

Interestingly, the influence of tropical South Atlantic and Gulf of Guinea SSTs on both regions changes as a function of emissions, shifting from a positive correlation at low emissions

390 to a neutral or negative correlation at high emissions. This suggests changing dynamical

391 interactions between remote forcings like ENSO and the more local influence of SSTs in the 392 neighboring Atlantic Ocean. It is also noteworthy that for region 2 there is an Atlantic Meridional 393 Mode (AMM) type signal in the historical simulations and RCPs 2.6 and 4.5, but this signal 394 disappears in RCP 8.5, where the cross-equatorial SST gradient is absent. This again points to 395 changing relationships between the tropical Atlantic Ocean and Sahel precipitation. We do note 396 that all results shown here are for a single GCM ensemble member, which is expected to impact 397 results for shorter time scales and might also impact the details of these 2006-2100 results.

\section{5. Conclusions}

The different clustering methods available in HiClimR can be useful for different 400 regionalization problems. Ward's method is sensitive to region size and tends to divide a large 401 homogeneous region into multiple separate regions. In contrast, the regional linkage method can 402 identify a big coherent region and filter out noisy data. For example, Ward's method was effective 403 in winter months, when regions have relatively similar size, while regional linkage yielded cleanly 404 separated and contiguous homogeneous regions in summer, when the interannual variability of 405 Sahel precipitation is dominant. Regional linkage also provides more climatically relevant results 406 in the transition seasons.

407 In the historical observational record, the Sahel expands from West to East and dominates 408 the interannual variability of African precipitation in summer. This is confirmed with the spatial 

correlation patterns of the region mean and precipitation over Africa using CHIRPS data. The

410 overall homogeneity of the region is only moderately high, but it is spread across the region with

411 highly significant correlation between any potentially identifiable subregions. This suggests that

412 interannual variability is characterized by one dominant mode of variability for the entire region 413 plus local modes of variability in the subregions. The winter months (December-March) are

414 relatively stable in their regionalization, and with a few exceptions can be treated as a coherent 415 season for climate analysis. As expected, the transitional months have complicated spatial 416 variability.

417 We tested the potential of climate regionalization for model intercomparision and 418 assessment. CCSM4 and MIROC5 showed good skill in capturing the precipitation signal over the 419 Sahel in summer, while the other models miss the spatial patterns by dividing the Sahel into smaller 420 regions with dissimilar interannual variability. CNRM generates random spatial patterns, GFDL 421 simulates Sahel-like precipitation variability in summer shifted to the northwest and HadGEM2 422 divides the Sahel region into eastern and western subregions. This does not mean that models like 423 CNRM, GFDL, or HadGEM2 are not useful for projecting climate change in Africa. On the 424 contrary, the regionalization analysis presented in this paper might allow us to interpret these 425 simulations properly for studies of climate process and projections of climate change impacts. If 426 GFDL systematically shifts the "Sahel" climate pattern to the northwest, then analysis of Sahel 427 sensitivity in this model should be performed with a corresponding shift. This opens the possibility 428 of a more meaningful, regionalization-based multi-model ensemble of climate projections for the 429 Sahel — or for any region in which models differ in the spatial representation of variability. In the 430 long term, these studies can also inform model development to correct spatial biases and 431 displacements in the representation of climate variability. 


\section{Acknowledgements}

433 We acknowledge the World Climate Research Programme's Working Group on Coupled 434 Modeling, which is responsible for CMIP, and we thank the climate modeling groups for 435 producing and making available their model output. For CMIP the U.S. Department of Energy's 436 Program for Climate Model Diagnosis and Intercomparison provides coordinating support and led 437 development of software infrastructure in partnership with the Global Organization for Earth 438 System Science Portals. Work for this paper was supported in part by NASA Applied Sciences 439 grant 13-WATER13-0010 and NSF's Dynamics of Coupled Natural and Human Systems (CNH)

440 Program award GEO-1211235. 
442 Badr, H. S., B. F. Zaitchik, and S. D. Guikema, 2014a: Application of Statistical Models to the

443 Prediction of Seasonal Rainfall Anomalies over the Sahel. Journal of Applied Meteorology and

444 Climatology, 53, 614-636.

445 Badr, H. S., B. F. Zaitchik, and A. K. Dezfuli, 2014b: HiClimR: Hierarchical Climate

446 Regionalization. Comprehensive R Archive Network (CRAN), [ Available online at http://cran.r-

447 project.org/package=HiClimR ].

$448 \longrightarrow$, 2015: A tool for hierarchical climate regionalization. Earth Science Informatics, 1-10.

449 Balas, N., S. Nicholson, and D. Klotter, 2007: The relationship of rainfall variability in West

450 Central Africa to sea-surface temperature fluctuations. International journal of climatology, 27, 451 1335-1349.

452 Barry, R. G., and R. J. Chorley, 2009: Atmosphere, weather and climate. Routledge.

453 Burn, D. H., 1989: Cluster analysis as applied to regional flood frequency. Journal of Water

454 Resources Planning and Management, 115, 567-582.

455 Dezfuli, A. K., 2011: Spatio-temporal variability of seasonal rainfall in western equatorial

456 Africa. Theoretical and applied climatology, 104, 57-69.

457 Dezfuli, A. K., and S. E. Nicholson, 2013: The Relationship of Rainfall Variability in Western

458 Equatorial Africa to the Tropical Oceans and Atmospheric Circulation. Part II: The Boreal

459 Autumn. Journal of Climate, 26.

460 Dickinson, R. E., 1995: Land-atmosphere interaction. Reviews of Geophysics, 33, 917-922.

461 Djomou, Z. Y., D. Monkam, and R. Chamani, 2015: Characterization of climatic zones, 462 variability and trend in northern Africa. Climate Dynamics, 44, 3481-3491.

463 Flohn, H., 1964: Investigations on the tropical easterly jet. Dümmlers Vlg. 
464 Funk, C., and Coauthors, 2015: The climate hazards infrared precipitation with stations - a new 465 environmental record for monitoring extremes. Scientific data, 2.

466 Gong, X., and M. B. Richman, 1995: On the application of cluster analysis to growing season 467 precipitation data in North America east of the Rockies. Journal of Climate, 8, 897-931.

468 Herrmann, S. M., and K. I. Mohr, 2011: A continental-scale classification of rainfall seasonality 469 regimes in Africa based on gridded precipitation and land surface temperature products. Journal 470 of Applied Meteorology and Climatology, 50, 2504-2513.

471 Huang, B., and Coauthors, 2015: Extended Reconstructed Sea Surface Temperature Version 4 472 (ERSST. v4). Part I: Upgrades and Intercomparisons. Journal of Climate, 28, 911-930.

473 Isik, S., and V. P. Singh, 2008: Hydrologic regionalization of watersheds in Turkey. Journal Of $474 \quad$ Hydrologic Engineering, 13, 824-834.

475 Janicot, S., 1992: Spatiotemporal variability of West African rainfall. Part I: Regionalizations 476 and typings. Journal of Climate, 5, 489-497.

477 Liu, W., and Coauthors, 2015: Extended Reconstructed Sea Surface Temperature Version 4 478 (ERSST. v4): Part II. Parametric and Structural Uncertainty Estimations. Journal of Climate, 28, $479931-951$.

480 Mahe, G., Y. L'hote, J. C. Olivry, and G. Wotling, 2001: Trends and discontinuities in regional 481 rainfall of West and Central Africa: 1951-1989. Hydrological Sciences Journal, 46, 211-226.

482 Moss, R. H., and Coauthors, 2010: The next generation of scenarios for climate change research 483 and assessment. Nature, 463, 747-756.

484 Murtagh, F., 1983: A survey of recent advances in hierarchical clustering algorithms. The 485 Computer Journal, 26, 354-359. 
486 Nicholson, S. E., 1986: The spatial coherence of African rainfall anomalies: interhemispheric 487 teleconnections. Journal of climate and applied meteorology, 25, 1365-1381.

488 Nicholson, S. E., and A. K. Dezfuli, 2013: The Relationship of Rainfall Variability in Western

489 Equatorial Africa to the Tropical Oceans and Atmospheric Circulation. Part I: The Boreal

490 Spring. Journal of Climate, 26.

491 Ogallo, L., 1989: The spatial and temporal patterns of the East African seasonal rainfall derived 492 from principal component analysis. International Journal of Climatology, 9, 145-167.

493 Ramachandra Rao, A., and V. Srinivas, 2006: Regionalization of watersheds by hybrid-cluster 494 analysis. Journal of Hydrology, 318, 37-56.

495 Ward Jr, J. H., 1963: Hierarchical grouping to optimize an objective function. Journal of the 496 American statistical association, 58, 236-244. 


\section{Tables}

498 Table 1 Intra-regional and maximum inter-regional correlations for each month....................25 
499 Table 1 Intra-regional and maximum inter-regional correlations for each month.

\begin{tabular}{|c|c|c|c|c|c|c|c|c|c|c|c|c|c|}
\hline \multirow{2}{*}{ MM } & \multicolumn{12}{|c|}{ Intra-regional Correlations $\left(R_{n n}{ }^{1}\right)$} & \multirow{2}{*}{$\mathbf{R}_{\mathrm{mx}}^{2}$} \\
\hline & $\mathbf{R}_{\mathbf{0 1}}$ & $\mathbf{R}_{02}$ & $\mathbf{R}_{\mathbf{0 3}}$ & $\mathbf{R}_{\mathbf{0 4}}$ & $\mathbf{R}_{05}$ & $\mathbf{R}_{06}$ & $\mathbf{R}_{\mathbf{0 7}}$ & $\mathbf{R}_{\mathbf{0 8}}$ & $\mathbf{R}_{09}$ & $\mathbf{R}_{10}$ & $\mathbf{R}_{11}$ & $\mathbf{R}_{12}$ & \\
\hline 01 & 0.71 & 0.49 & 0.66 & 0.61 & 0.61 & & & & & & & & 0.48 \\
\hline 02 & 0.72 & 0.51 & 0.68 & 0.64 & 0.53 & & & & & & & & 0.43 \\
\hline 03 & 0.59 & 0.49 & 0.74 & 0.60 & 0.63 & 0.62 & & & & & & & 0.42 \\
\hline 04 & 0.57 & 0.72 & 0.66 & 0.67 & 0.73 & 0.87 & 0.70 & 0.74 & 0.57 & 0.72 & 0.67 & 0.73 & 0.49 \\
\hline 05 & 0.54 & 0.67 & 0.46 & 0.64 & 0.63 & 0.57 & 0.69 & & & & & & 0.49 \\
\hline 06 & 0.54 & 0.46 & 0.50 & 0.57 & & & & & & & & & 0.35 \\
\hline 07 & 0.60 & 0.58 & 0.54 & 0.66 & & & & & & & & & 0.39 \\
\hline 08 & 0.58 & 0.61 & 0.62 & 0.60 & 0.59 & & & & & & & & 0.42 \\
\hline 09 & 0.52 & 0.56 & 0.54 & 0.59 & 0.61 & & & & & & & & 0.36 \\
\hline 10 & 0.69 & 0.67 & 0.64 & 0.68 & 0.56 & 0.82 & 0.72 & 0.64 & 0.73 & 0.70 & 0.80 & & 0.51 \\
\hline 11 & 0.54 & 0.68 & 0.70 & 0.60 & 0.63 & 0.67 & 0.68 & 0.70 & & & & & 0.44 \\
\hline 12 & 0.58 & 0.47 & 0.73 & 0.57 & 0.67 & 0.68 & & & & & & & 0.33 \\
\hline
\end{tabular}

\footnotetext{
${ }^{1} R_{n n}$ is the average intra-regional correlation for region number nn, between the region mean and all members within the region.

${ }^{2} R_{m x}$ is the maximum inter-regional correlation between region means.
} 
502 Fig. 1 The effect of geographic masking and data filtering on the quality of regionalization: A) no masking, B) geographic masking of Africa, and C) geographic masking and data

Fig. 2 The effect of geographic masking and data filtering on the clustering dendrogram: A) no masking, B) geographic masking of Africa, and C) geographic masking and data filtering. 29

Fig. 3 The effect of detrending and standardization on regionalization quality: A) raw data and

Fig. 4 The effect of detrending and standardization on the clustering dendrogram: A) raw data B) detrended and standardized data.

Fig. 5 Regionalization based on: A) interannual variability of annual totals of precipitation and

514 Fig. 7 Regionalization of Africa based on interannual variability of Summer (JAS) precipitation

516 Fig. 8 Regionalization of Africa based on interannual variability of JAS precipitation in 1960-

518 Fig. 9 Regionalization of Africa based on interannual variability of JAS precipitation in 1981-

520 Fig. 10 Correlation patterns of CHIRPS precipitation with global ERSST for the mean timeseries

521 of the four regions of interannual variability of JAS precipitation at the period (1981-2014). 
523 Fig. 11 Correlation patterns of CCSM4 precipitation with the model SST for the mean timeseries 524 of the four regions of interannual variability of JAS precipitation at the period (1960-1990). All correlations are significant at $90 \%$ confidence level. 38

526 Fig. 12 Changes in the regions of JAS precipitation over Africa using regional-linkage method

Fig. 13 Correlation patterns of CCSM4 JAS precipitation from the four RCPs with the for different CCSM4 climate projections at the entire simulation period (2006-2100)........ 39 corresponding SST for region 1 (Western Sahel in Fig. 12) at the period (2006-2100). All correlations are significant at $90 \%$ confidence level.

531 Fig. 14 Correlation patterns of CCSM4 JAS precipitation from the four RCPs with the corresponding SST for region 2 (Eastern Sahel in Fig. 12) at the period (2006-2100). All 


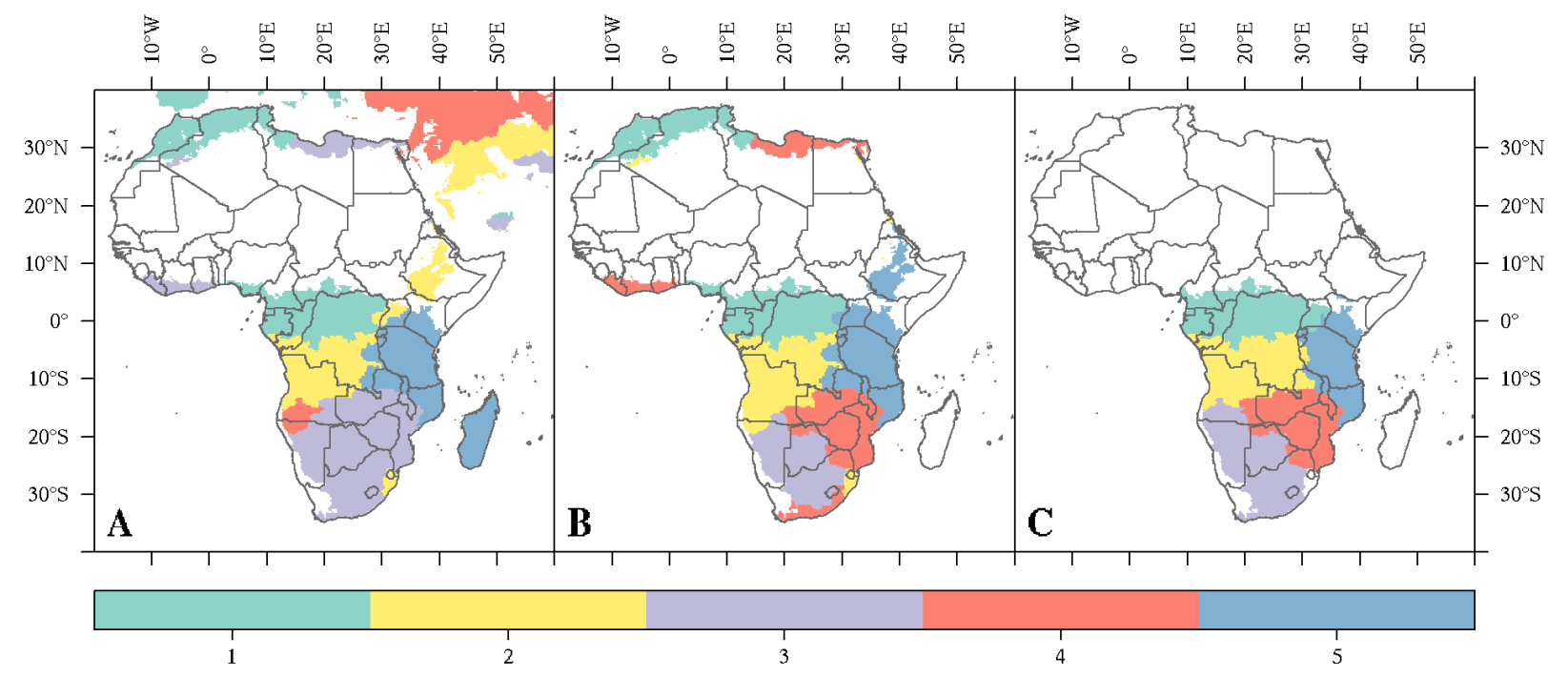

535 Fig. 1 The effect of geographic masking and data filtering on the quality of regionalization: A)

536 no masking, B) geographic masking of Africa, and C) geographic masking and data filtering. 


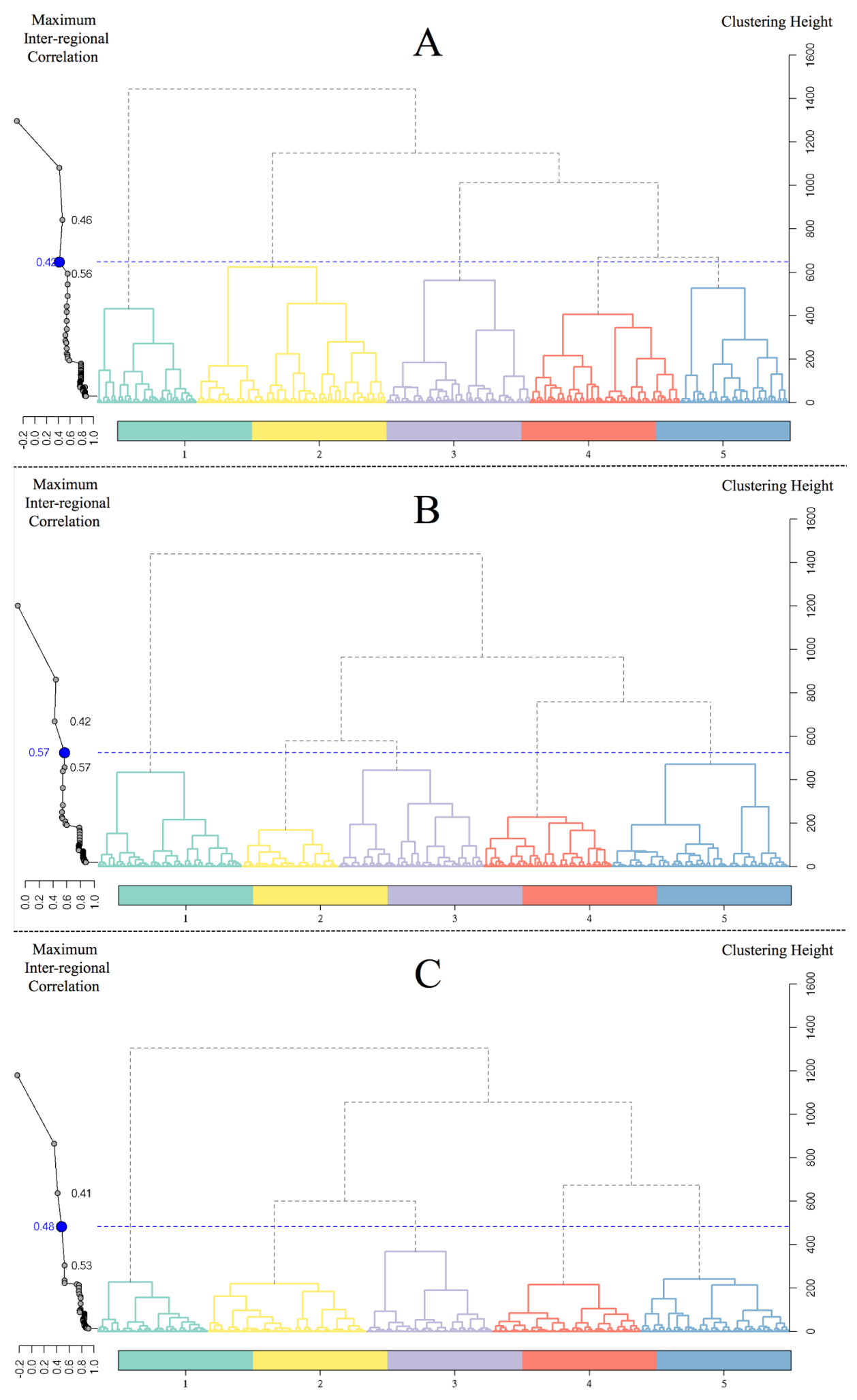

538 Fig. 2 The effect of geographic masking and data filtering on the clustering dendrogram: A) no 539 masking, B) geographic masking of Africa, and C) geographic masking and data filtering. 


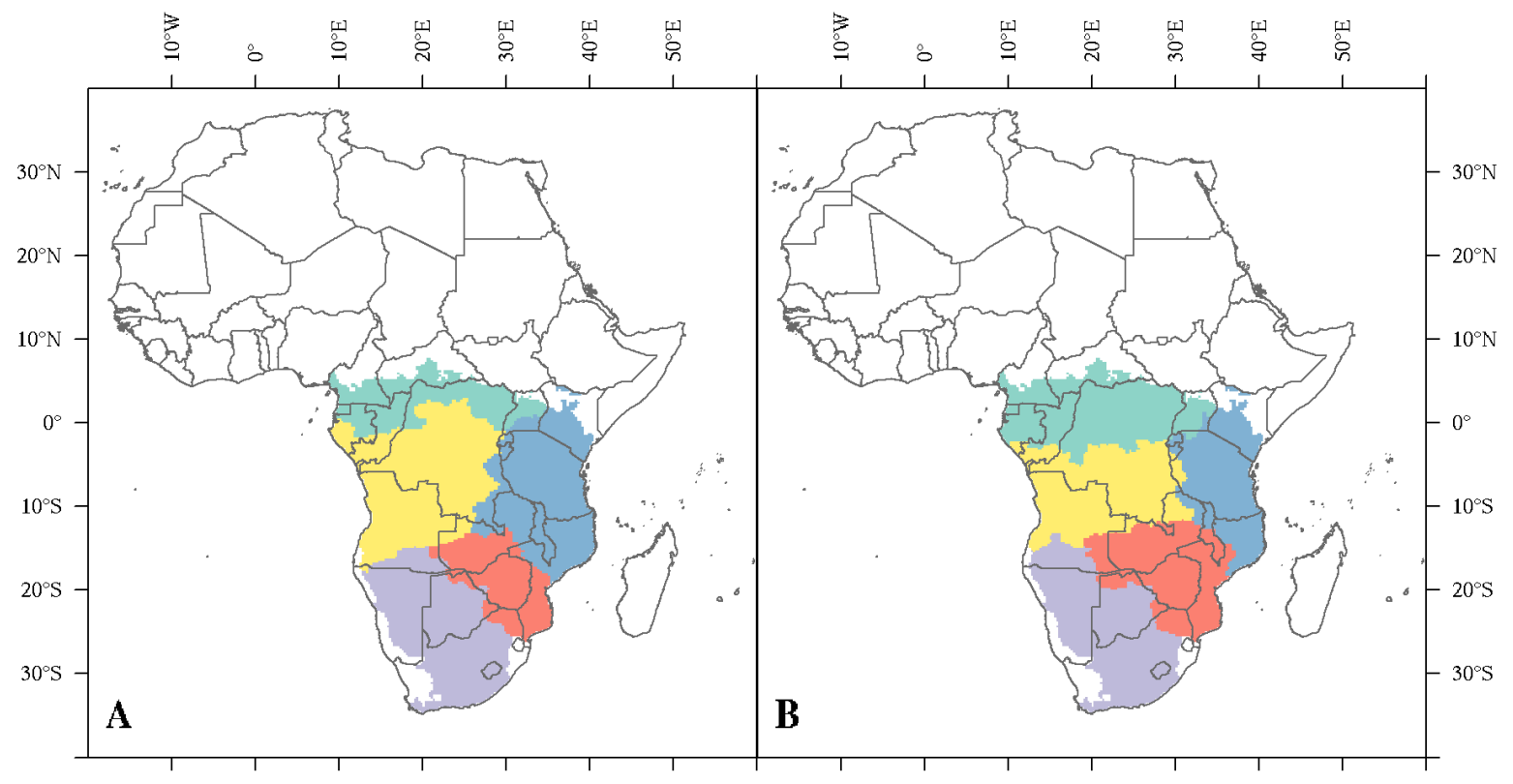

540

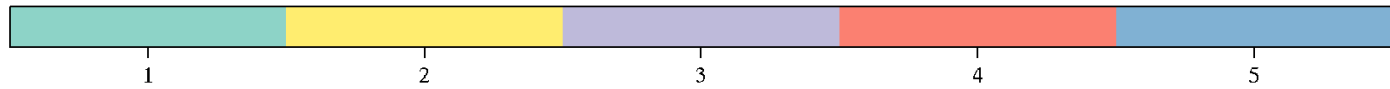

541 Fig. 3 The effect of detrending and standardization on regionalization quality: A) raw data and B) detrended and standardized data. 

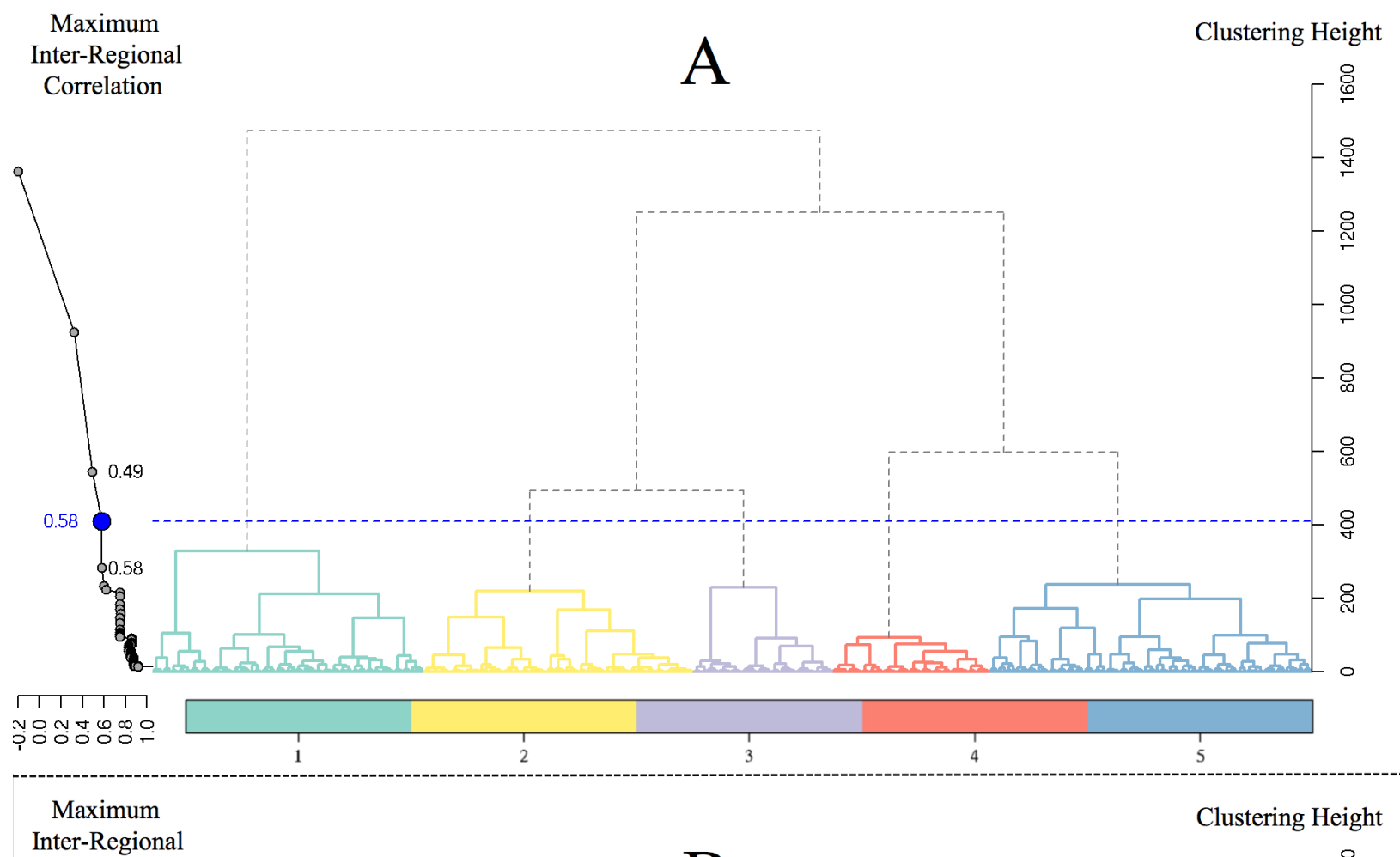

Correlation

$\mathrm{B}$

总

\&

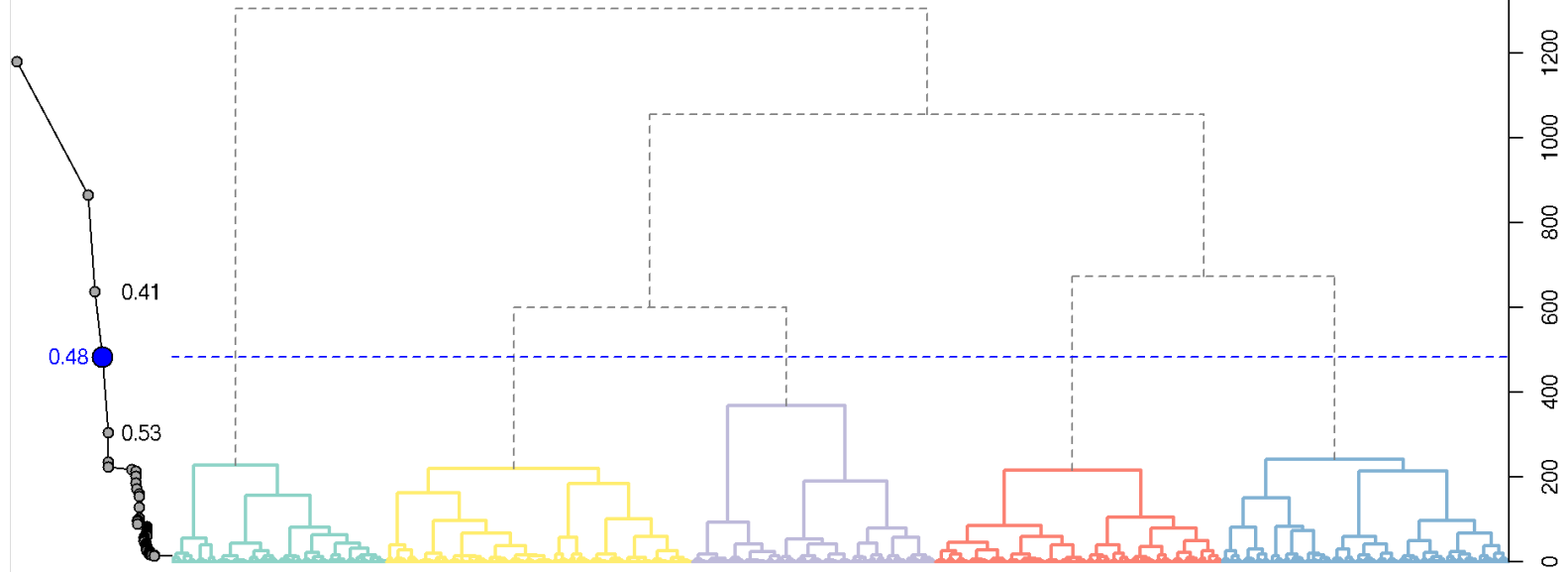

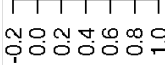

544 Fig. 4 The effect of detrending and standardization on the clustering dendrogram: A) raw data

545 and B) detrended and standardized data. 

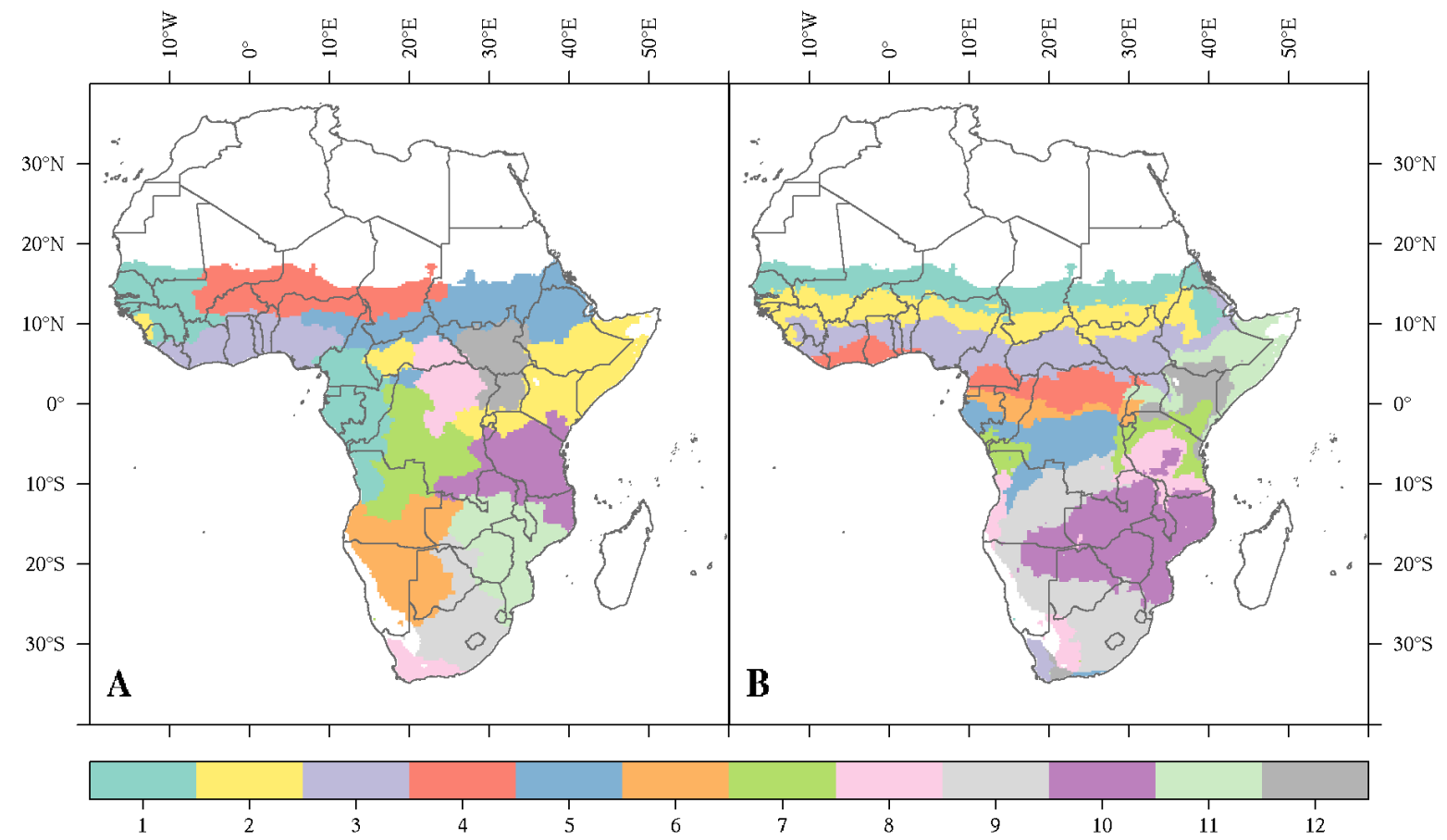

547 Fig. 5 Regionalization based on: A) interannual variability of annual totals of precipitation and B) annual cycle of precipitation over Africa using CHIRPS data v2.0 (1981-2014). 


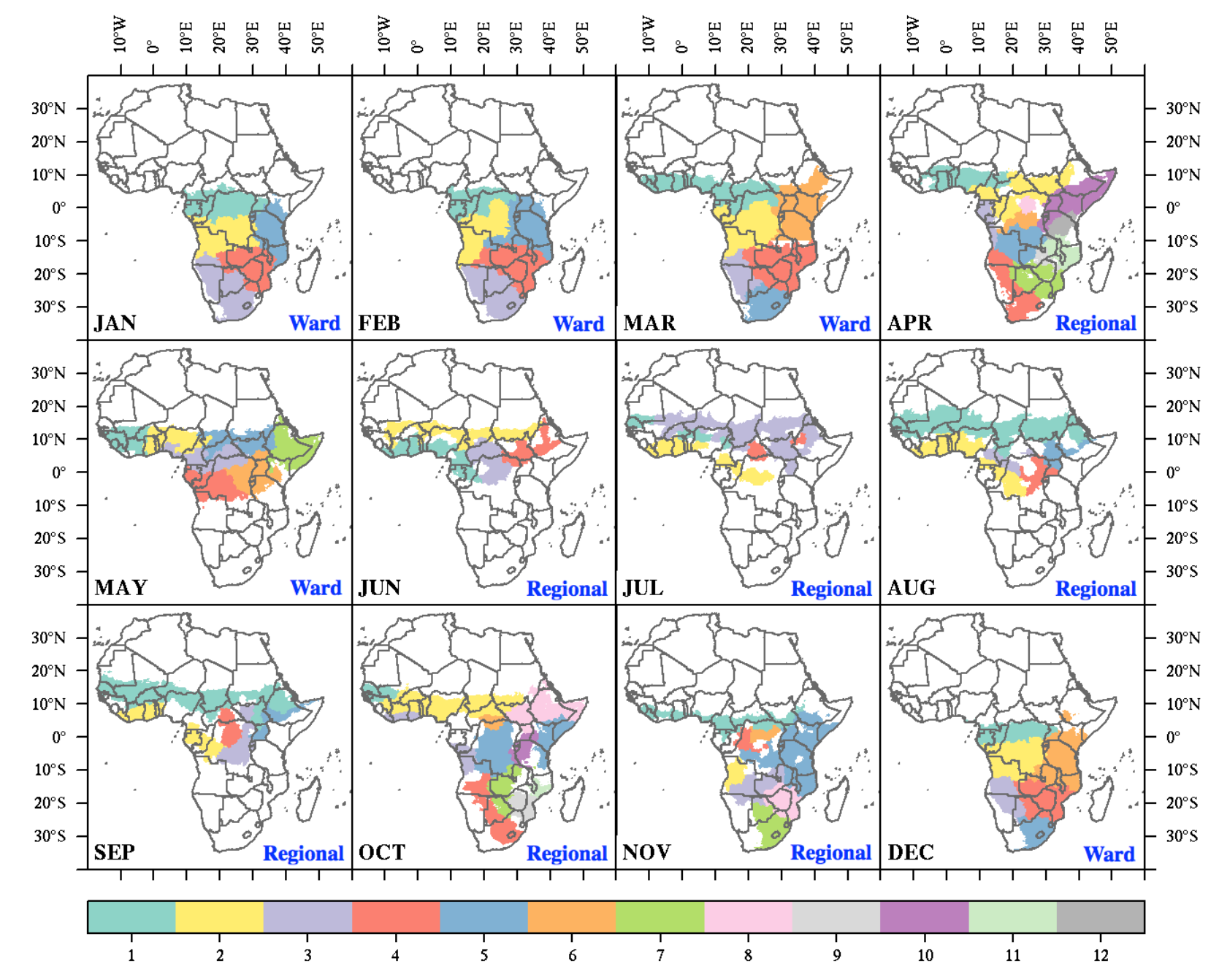

Fig. 6 Regionalization of Africa based on interannual variability of monthly precipitation. 


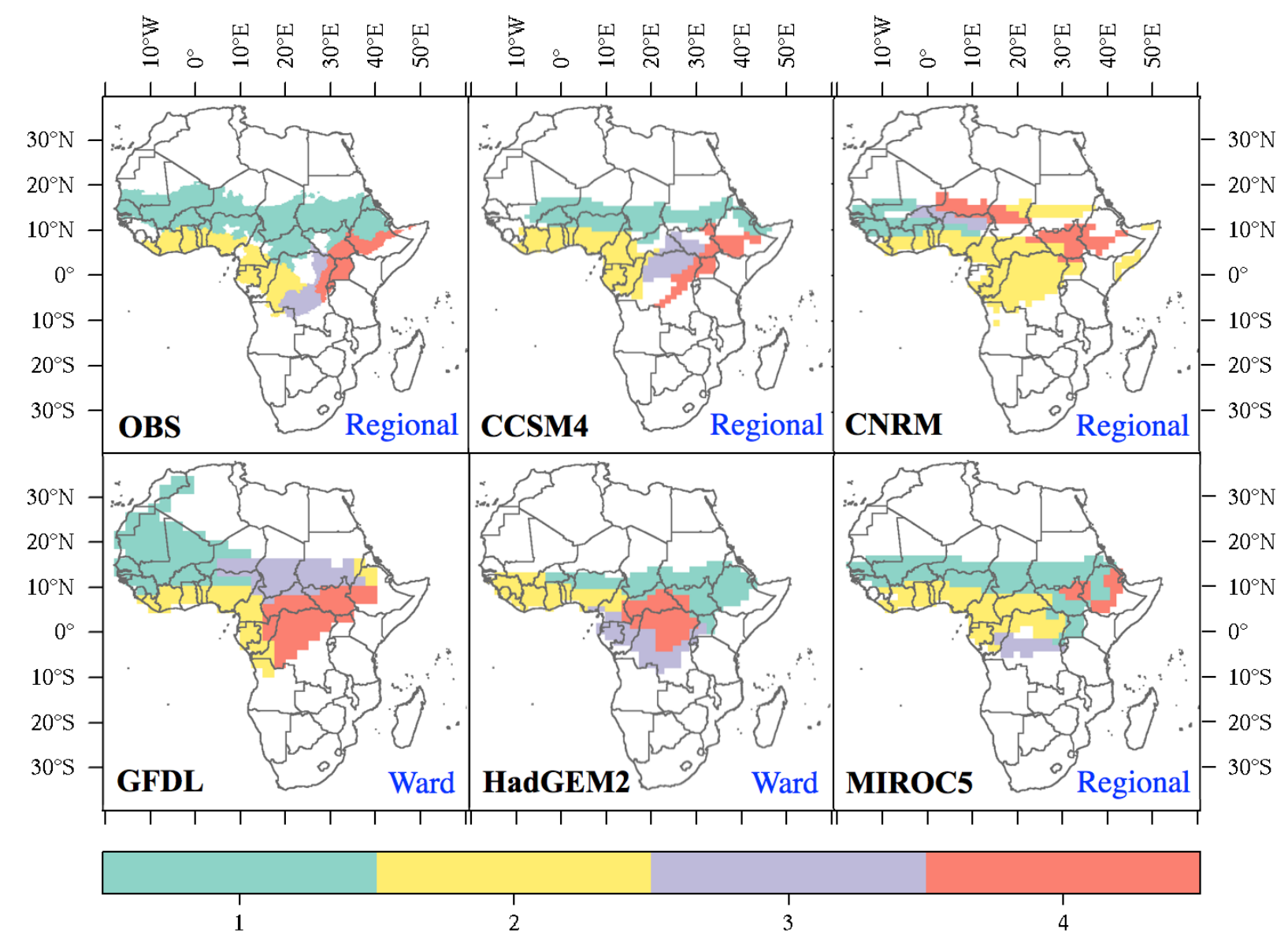

Fig. 7 Regionalization of Africa based on interannual variability of Summer (JAS) precipitation

553 using CHIRPS observations (1981-2014) and different GCMs (1960-1990). 


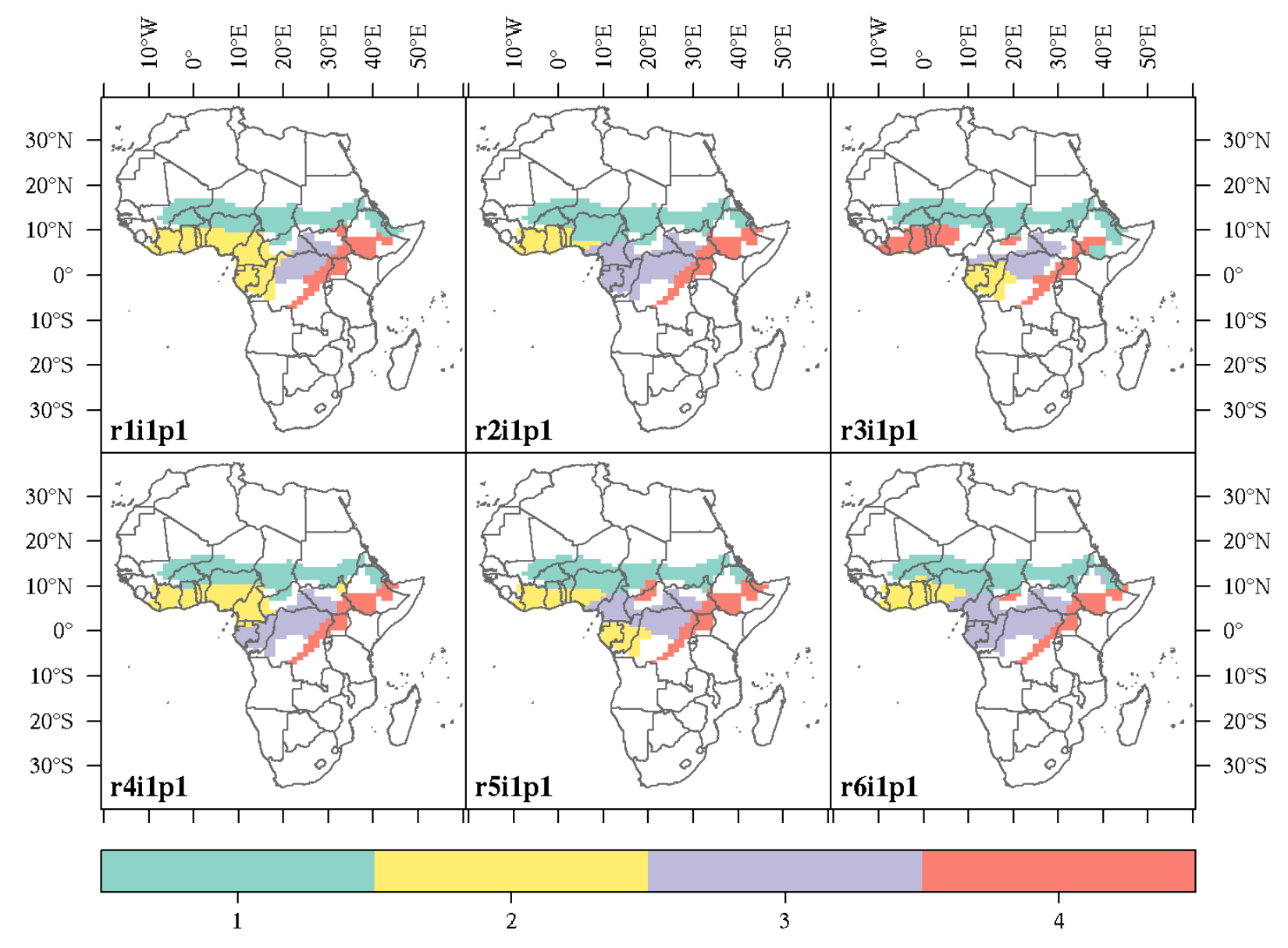

555 Fig. 8 Regionalization of Africa based on interannual variability of JAS precipitation in 1960-

5561990 for different ensemble members of CCSM4. 


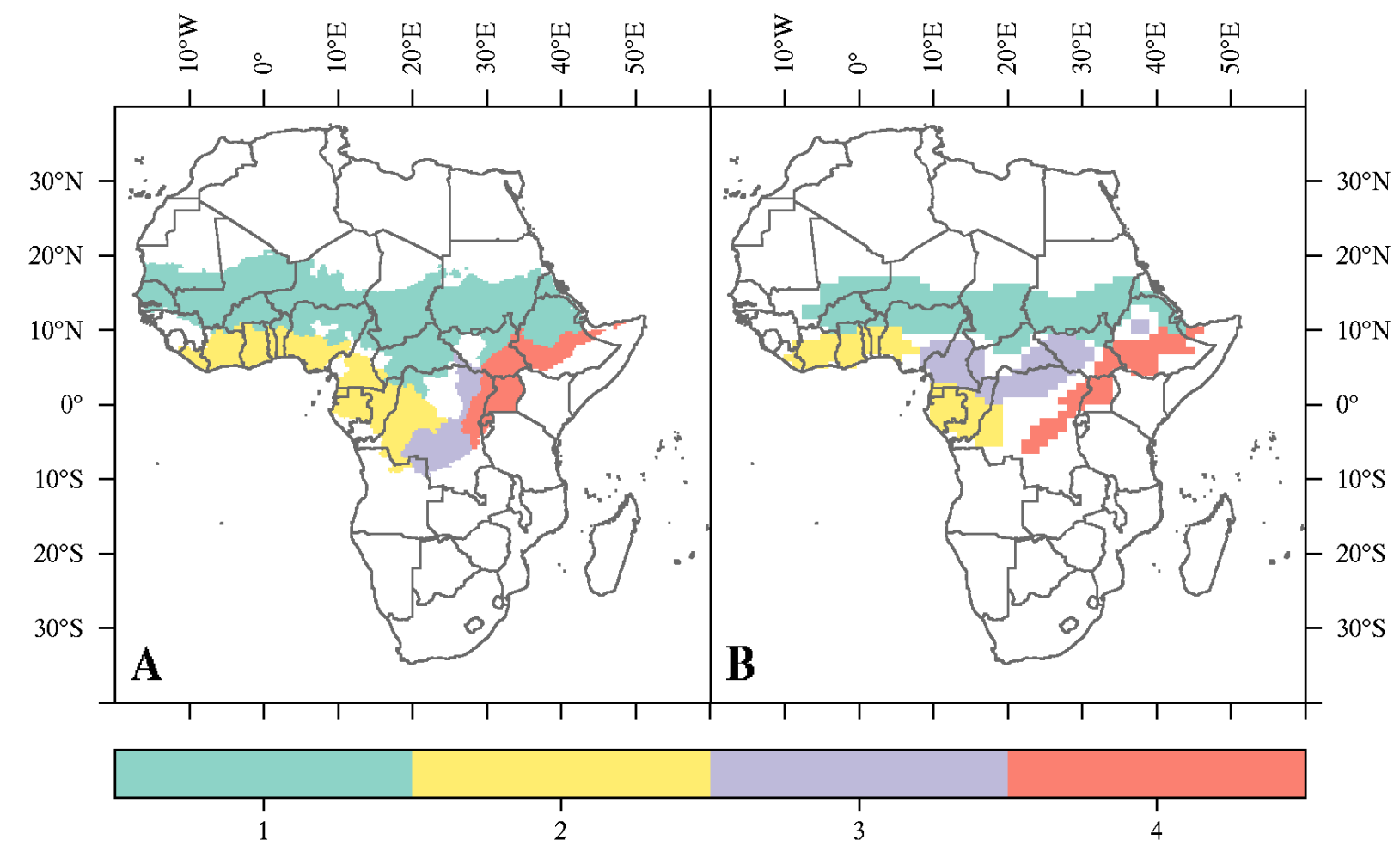

559 Fig. 9 Regionalization of Africa based on interannual variability of JAS precipitation in 1981-

5602014 using regional-linkage method: A) CHIRPS and B) CCSM4. 


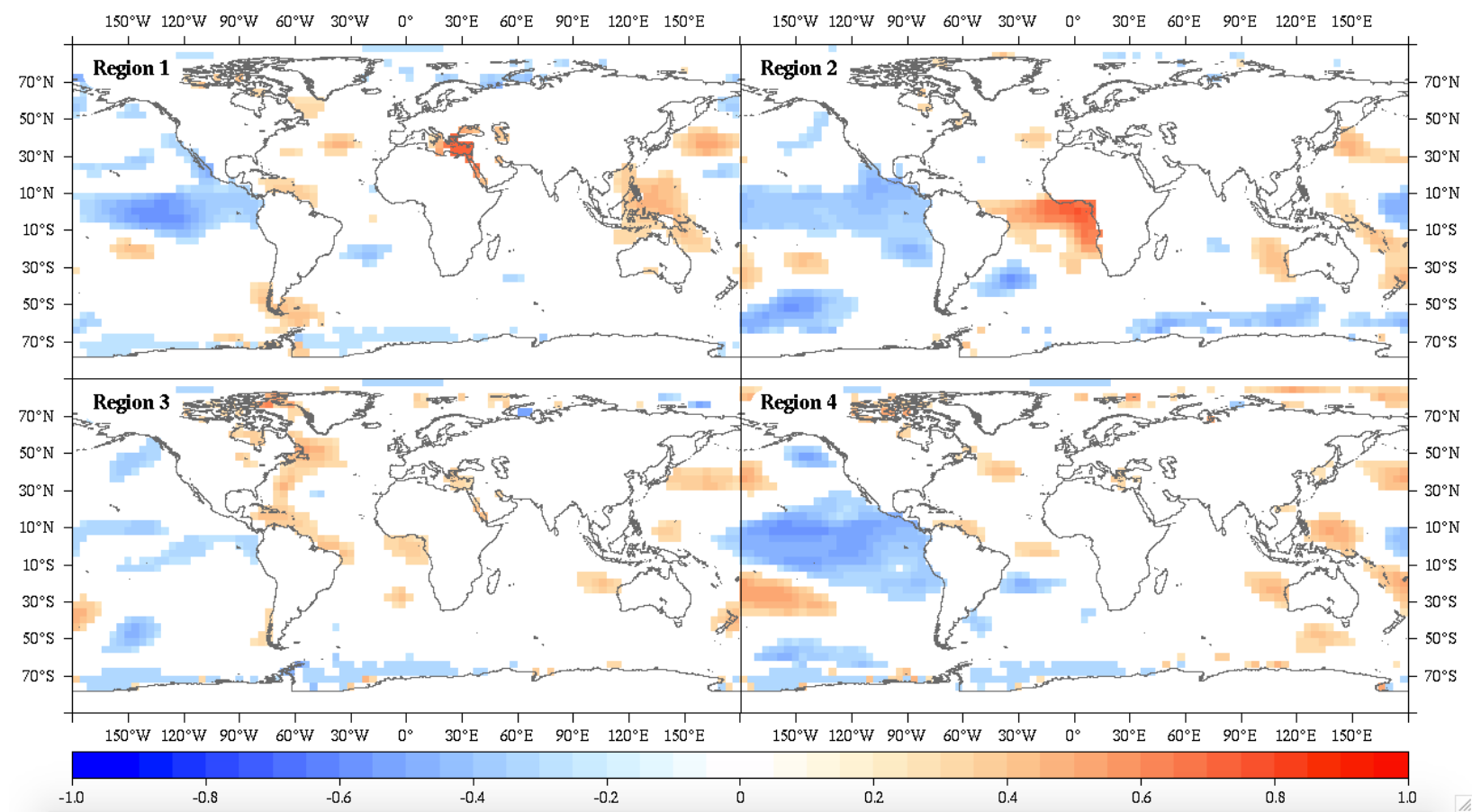

562 Fig. 10 Correlation patterns of CHIRPS precipitation with global ERSST for the mean timeseries

563 of the four regions of interannual variability of JAS precipitation at the period (1981-2014). All

564 correlations are significant at 90\% confidence level. 


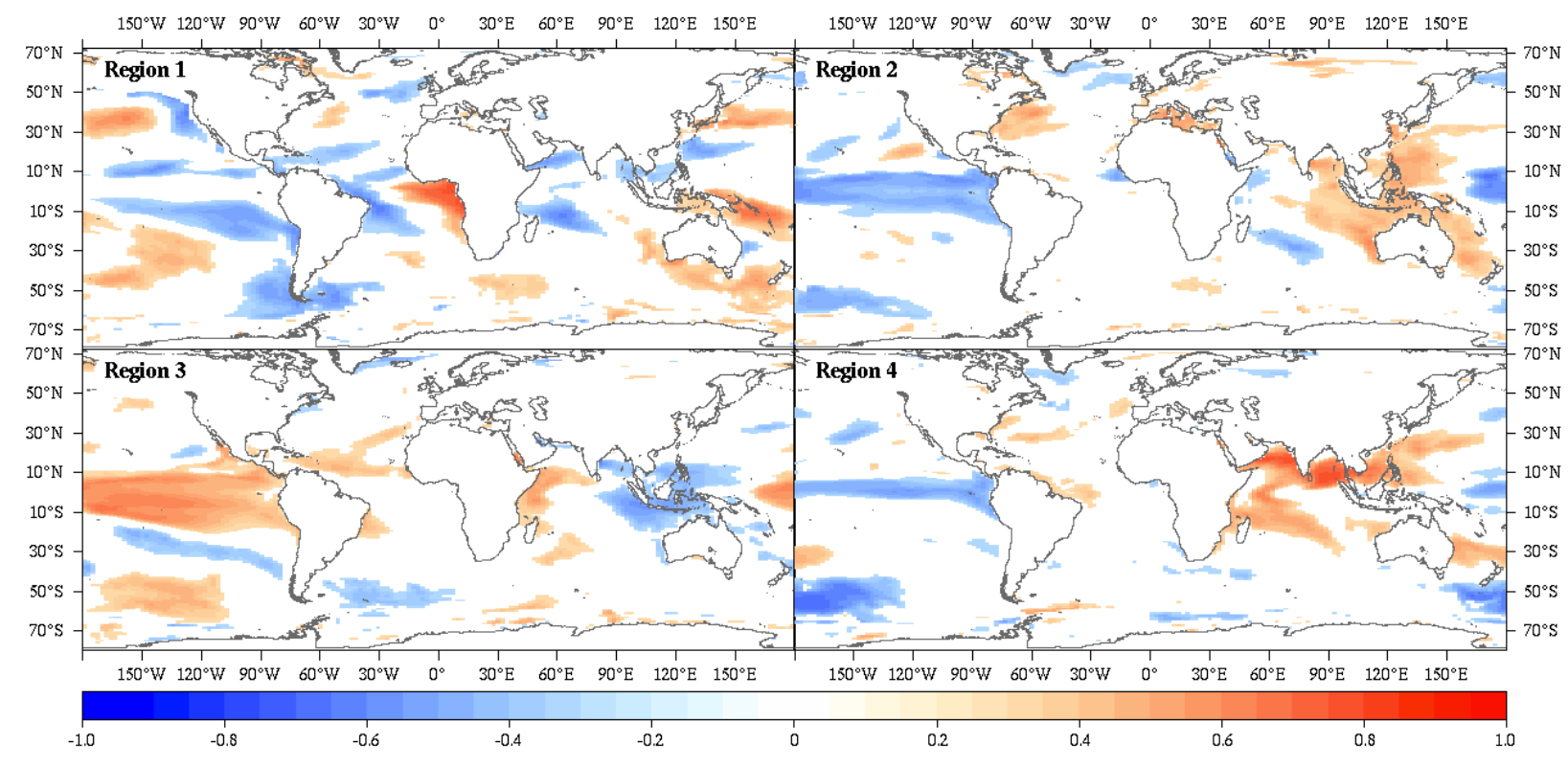

Fig. 11 Correlation patterns of CCSM4 precipitation with the model SST for the mean timeseries

567 of the four regions of interannual variability of JAS precipitation at the period (1960-1990). All

568 correlations are significant at $90 \%$ confidence level. 


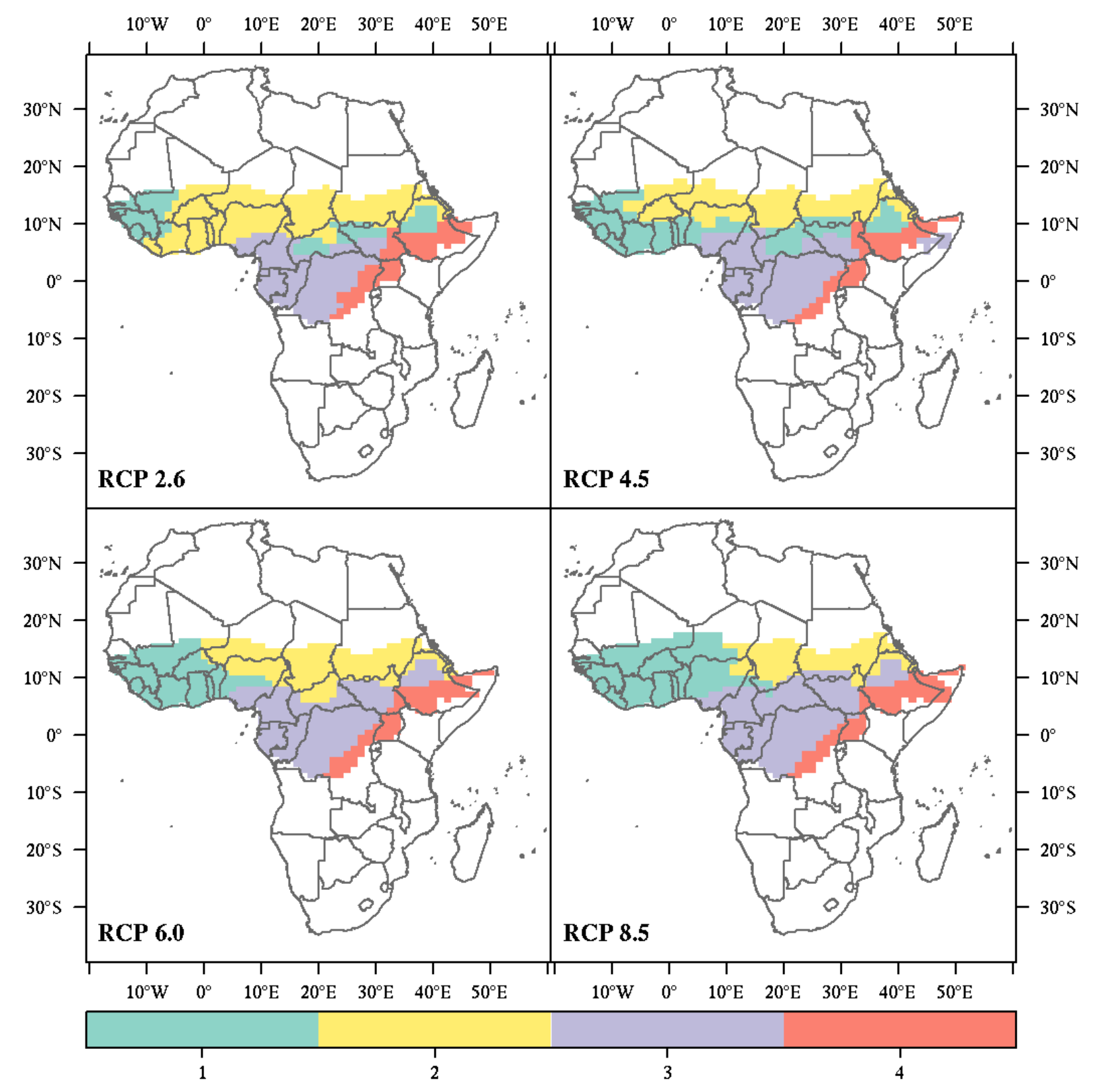

570 Fig. 12 Changes in the regions of JAS precipitation over Africa using regional-linkage method

571 for different CCSM4 climate projections at the entire simulation period (2006-2100). 


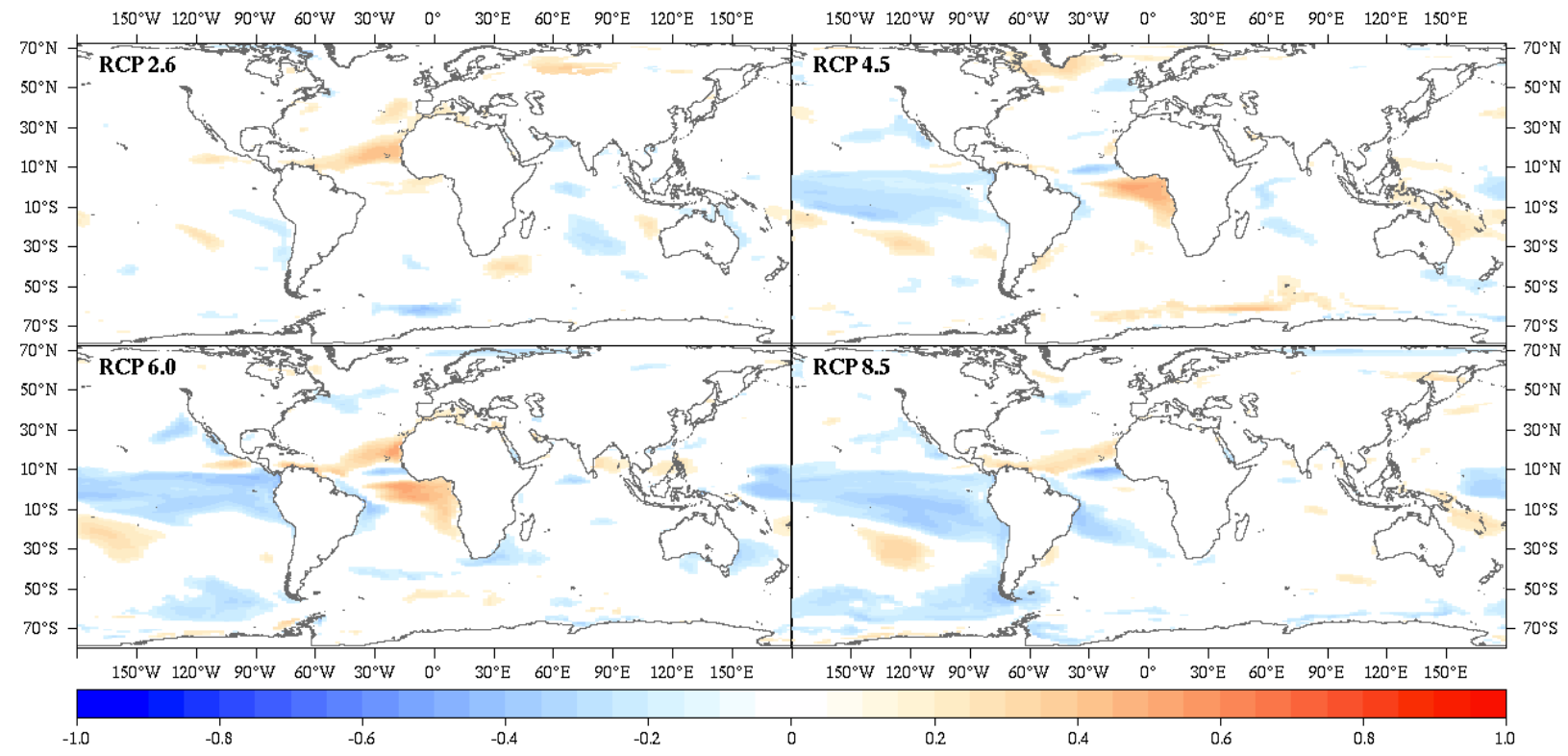

573 Fig. 13 Correlation patterns of CCSM4 JAS precipitation from the four RCPs with the

574 corresponding SST for region 1 (Western Sahel in Fig. 12) at the period (2006-2100). All

575 correlations are significant at $90 \%$ confidence level. 


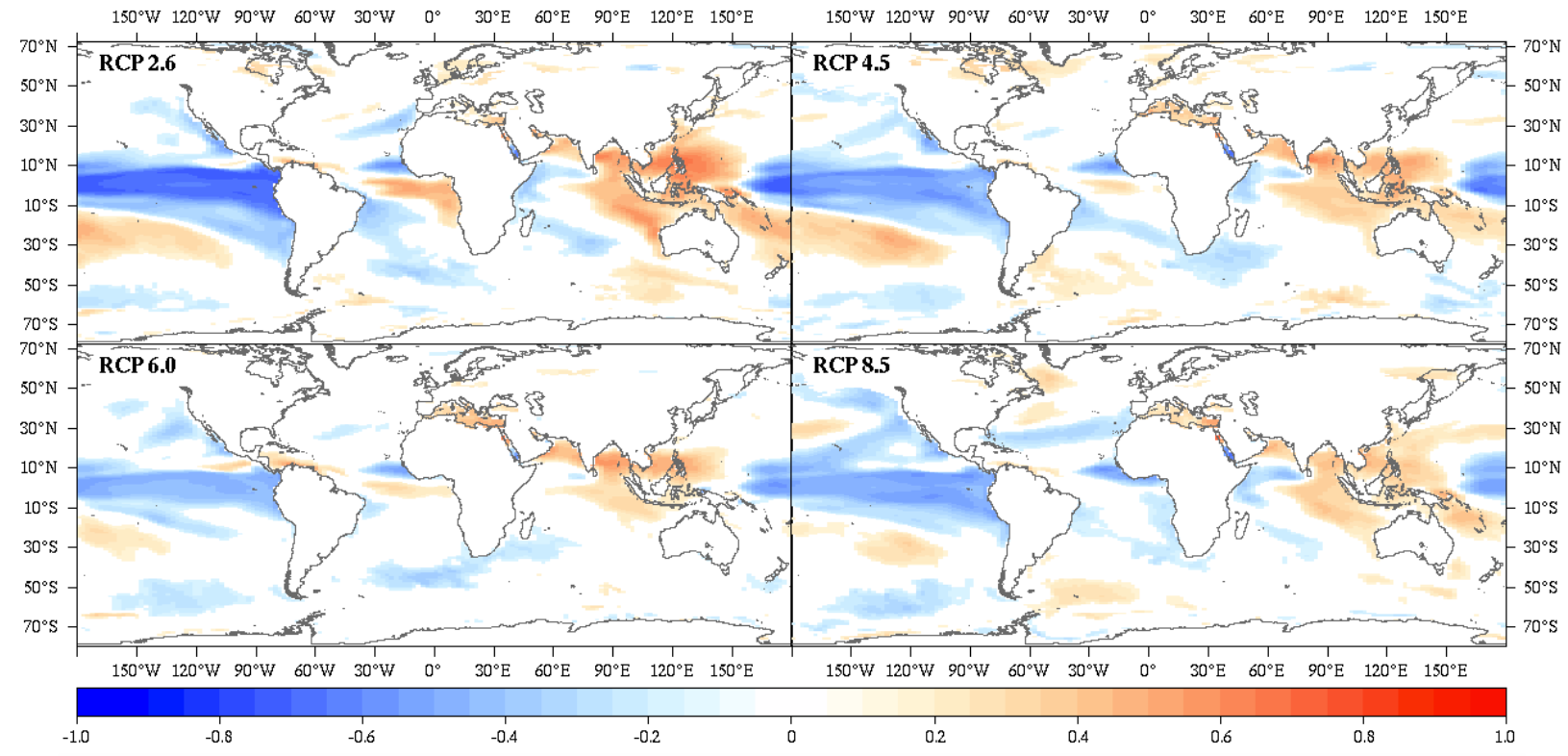

577 Fig. 14 Correlation patterns of CCSM4 JAS precipitation from the four RCPs with the

578 corresponding SST for region 2 (Eastern Sahel in Fig. 12) at the period (2006-2100). All

579 correlations are significant at $90 \%$ confidence level. 